\title{
Challenges for Integrated Design and Delivery Teams in AEC
}

\author{
Vishal Singh \\ Department of Civil and Structural Engineering, Aalto University, Finland \\ Vishal.Singh@aalto.fi
}

\begin{abstract}
This paper discusses the critical factors for managing teams for integrated design and delivery solutions (IDDS), which aims to involve BIM tools, processes and skills across different stakeholders such as architects, consultants, contractors, and suppliers through the project lifecycle. Since IDDS is a recent development, the associated teamwork factors and challenges in implementation are not currently well understood. This paper reports the findings from a research that investigates this gap and forms the basis for future research directions. The findings reported in this paper are based on focus group interviews with representatives from leading organizations in the Australian and Finnish construction sector. The critical factors for an IDDS team are examined and discussed using the construct of mental models. In particular, this research investigates the critical task, process, context, and competence factors specific to the IDDS teams.
\end{abstract}

Keywords: BIM, teamwork, mental models, integrated design and delivery solutions.

\section{Introduction}

The lack of integrated design and delivery across the lifecycle of multi-organizational construction projects and inefficient manpower management through the project lifecycle costs billions of dollars in waste [1]. As a result, there is a greater push for new ways of working that promote collaboration and integration across the project partners through the different phases of the project lifecycle. Accordingly, approaches such as Integrated Design Delivery Solutions (IDDS) and Integrated Project Delivery (IPD) are being developed, with greater emphasis on creating more productive environments through concurrent engineering [2], enabled by information systems, technology mediated practices, and collaborative work environments [3, 4]. This trend is based on the rationale that effective service integration and collaboration can reduce inefficiencies and waste, through reduced duplicity and rework [5, 6].

Nonetheless, full service and process integration is challenging and requires effective change management strategies [7, 8] and people engagement processes [9]. Hence, the change towards integrated design and delivery within the architecture, engineering and construction (AEC) sector is contingent on sound understanding of the teamwork and engagement processes. 
In the AEC sector, design integration requires a multiplicity of skills, knowledge and experience. Design practice requires management tools and skills besides the design skills and the domain knowledge. The performance of the collaborative design team not only depends on how the individual members perform the tasks related to their roles but also on how effectively they coordinate their tasks, roles and responsibilities with the other members of the team [2]. For collaborative teams to be efficient, team members need well developed mental models of each other and that of the task, process, context and competence of the team [10-13]. Well-developed mental models of the team members can significantly improve the cost, schedule, and quality measures, especially in complex projects [14], which is often the case with construction projects. Managing integrated construction project teams further increases the complexity as the team is expected to work concurrently across the project lifecycle. However, there is little research and understanding of the teamwork management issues within such teams, especially there is little structured breakdown of issues according to the task, process, context, competence and team factors that constitute the mental models of the members of the multi-organizational construction projects.

This paper reports on the findings from a research study aimed at identifying the critical factors affecting the formation of mental models in multi-organizational construction projects, especially in view of the IDDS objectives. In particular, we investigate the critical task, process, context and competence factors specific to the integrated design and delivery teams.

The reported findings are based on focus group interviews (FGIs) and face-to-face (FFs) interviews with representatives from Australian and Finnish AEC sector on the potential challenges to integrated design delivery practice using a collaboration platform, with Building Information Model (BIM) at the core of data exchange.

\section{Background}

The construction sector is known to be a slow adopter of management and technical capabilities that other industries such as manufacturing are able to integrate earlier in their practice. Among others, the structural organization of the construction sector, which is markedly different to that of the manufacturing sector, is mooted as an important factor [15]. As a result, integration of key players is considered to be important for project success. As each player pursues its own contractual objectives, it is suspected that a lack of integration and teamwork has resulted in low productivity and low innovation. Such challenges are likely to impede the IDDS initiatives envisioned by the construction industry as the design and delivery practice of the future. One of the biggest challenges for managers and organizations involved in the multi-organizational design and construction projects is to understand, and deal with, a distributed project team with diverse task, process, context, and team member mental models, resulting from the diversity in social, operational and functional backgrounds of the team members at individual as well as organizational level. 
Formation of mental models has been studied across diverse domains [10, 16]. It is well established that effective teamwork requires various kinds of competencies that can be discussed in terms of the knowledge, skills and attitudes that are specific or generic to the task, and the team [17-19]. Mental models for the task, process, context, competence and the team members are critical to effective team performance [10-13]. These findings suggest that organizational contingency theories [20] also apply to formation of mental models in teams. That is, the critical parameters associated with the different mental models and their values may be specific to the project, despite generic patterns across an industry. Therefore, specific parameters and their values need to be mapped for each project, Table1.

Table 1. Mental models that team members need to develop

\begin{tabular}{ll}
\hline Mental model & Kinds of information that it requires (examples) \\
\hline Task & What is the task? What comes next? \\
Process & How to do this? \\
Context & What to do in this situation? \\
Competence & Can we do this? Do we have the capability? \\
Team members & Who knows what? \\
\hline
\end{tabular}

Understanding the likely effects of pre-developed mental models of team members, based on their prior experience, and the impending project requirements, can improve project decision making. Such approaches have been used to form effective small project teams, where the scale of the project and the personnel data is manageable using a manual process. For example, [21] reports the use of psychometric data to consistently form high performing student design teams. However, in real world construction projects, the complexity, the number of variables, and the amount of data to analyze can be expected to be much higher. This means such approaches towards creating high performance teams cannot be adopted unless decision support systems are developed to record, manage and simulate large data sets.

This research is part of a research initiative that aims to address some of the related issues. The critical factors pertaining to teamwork in IDDS projects identified in this research will contribute towards the development of such a decision support system (DSS). The planned agent-based DSS will build on the prior research on computational modeling of project teams $[22,23]$.

\section{Research Data Collection and Analysis}

The findings reported in this paper are based on data from FGIs and FFs conducted with representatives from various stakeholder groups that typically form an IDDS team. Three FGIs were conducted in two major cities in Australia and included architects, engineers, consultants, contractors, facility managers and construction project collaboration platform service providers. Representatives from large contracting firms and government organizations, who often work as clients in construction projects also participated in the workshop. The FGI discussions revolved 
around the potential challenges to integrated design delivery practice using a collaboration platform, with BIM at the core of data exchange. The FGI participants were encouraged to discuss the challenges to using an integrated information system and Building Information Models for managing the information supply chain across the project lifecycle. The FGI data was complemented by twelve FFs, two in Australia and ten in Finland. The FGIs and FFs were recorded on tape and transcribed for analysis to categorize the discussions across the issues relating to task, process, context, competence and team member mental models.

Verbal protocol analysis technique was used to analyze the Australian data and identify the underlying patterns and trends across the identified themes, i.e., task, process, context, competence and team. Table 2 provides sample segments to exemplify the classification and the coding scheme. "Protocol analysis is a rigorous methodology for eliciting verbal reports of thought sequences as a valid source of data on thinking" [24].

Preliminary analysis of part of the Finnish data has been conducted, and verbal protocol analysis of the Finnish data is yet to be conducted.

Table 2. Sample coding of data to classify factors across different mental models

\begin{tabular}{|c|c|c|c|c|c|}
\hline Segment & Task & Process & Context & Competence & Team \\
\hline $\begin{array}{l}\text { "Consultants and engineers tend to } \\
\text { work in isolation..." }\end{array}$ & & & & & 1 \\
\hline $\begin{array}{l}\text { "Clash detection, ability to check } \\
\text { dimensions, approval... all sort of } \\
\text { things should come out of the } \\
\text { database." }\end{array}$ & & 1 & & 1 & \\
\hline $\begin{array}{l}\text { "Important to understand what } \\
\text { record has to be kept... Can be a } \\
\text { photo, snapshot of what is on screen, } \\
\text { copy of contract document ...it is not } \\
\text { about the model, it is about how you } \\
\text { want to maintain that record." }\end{array}$ & & & & & \\
\hline $\begin{array}{l}\text { "This is going to change the way we } \\
\text { work. At an early stage we need a lot } \\
\text { more communication." }\end{array}$ & & 1 & 1 & & \\
\hline
\end{tabular}

\section{$4 \quad$ Research Findings}

Figure 1 shows the discussion pattern in the FGIs, based on the verbal protocol analysis results. As plotted in Figure1, though the data indicate a fairly uniform distribution of issues and concerns associated with the different mental models, i.e, task, process, context, competence and team members, the apprehensions around processes, including work and business processes is relatively higher. 


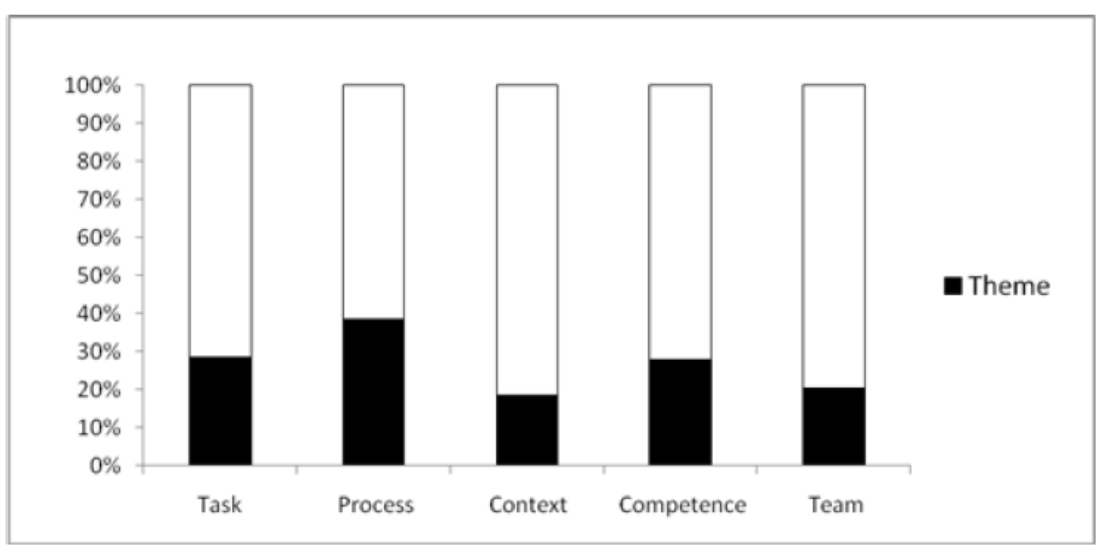

Fig. 1. Discussion pattern in FGIs reflected by $\%$ of segments dedicated to each theme (sum is greater than $100 \%$ as many issues concerned more than one theme)

The open ended discussions primarily revolved around the challenges in developing and using an integrated Building Information Model in a project, and the issues with managing the information. Discussions indicate that even at the outset of a project the different stakeholders are likely to start with conflicting mental models and apprehensions about each other's beliefs and level of engagement in the project. Following are some of the key factors associated with the task, process, context, and competence and team member mental models:

\subsection{Task Mental Model}

The integrated project team is expected to collectively work together in providing timely information, feedback and data through a collaborative platform to create a detailed BIM for the project, which many interviewees considered as central to the task definition in an IDDS project. Following are the critical task mental model related factor:

Purpose and Scope of the Model: Team members need a shared understanding of the purpose of BIM in the project. In some projects it might be desirable to create and use a BIM model across the entire project lifecycle while in other cases such models may only be developed for specific purposes such as simulating and planning constructability.

Level of Detail: Team members need some clarity on questions such as: How much detail is needed in the model? Which parts of the model need more details or do all parts of the model need the same level of details? How are the details at different levels of abstraction, and across the different phases of the project, to be linked and managed? Level of detail in the model is contingent on the project requirements and the purpose of the model. Clarity on the level of detail is important to determine work breakdown and planning. 
Structure of the Model: The use of BIM and the task typically requires decomposition and integration of the task. That is, model development is a multi-user activity, which means the team members need to coordinate the model development activities. Hence, the team members need to understand the task breakdown structure, and how the task is shared and distributed across the team.

Purpose and the level of the model needs to be clearly stated upfront as part of the contractual agreements. Findings indicate that the different project partners may have different expectations from the purpose as well as the level of detail desired in a BIM model. For example, designers, contractors and facility managers (FM) each view the purpose of the BIM model differently, and to each of these actors, the primary role of BIM is associated with their discipline. As such, each of these models such as the design model, construction model and FM model require different types, and different levels, of details. Therefore, an initial plan is needed on how these different models can be achieved through minimal rework and duplication, and maximum reuse.

Related aspects of task or model development where shared understanding is needed include model types and sub-models, accuracy and completeness, as-built data for facilities management, and documentation and communication tasks.

\subsection{Process Mental Model}

The Participants identified various aspects of the collaborative process that need discussion and clarity among team members and collaborators for effective management of an integrated BIM project development. This includes agreed protocols and practices for business processes, collaboration and information exchange, and interaction with the model. Mutual agreement and processes are critical for the following:

Business Processes: IDDS and IPD approaches are yet to be embraced in the construction industry and the lack of precedence and familiarity leaves apprehensions about business models and transactions. Among other concerns, FGI participants were unclear about the fee structures in the resulting re-configured work-load distribution and information supply chain; data transfer and ownership; and liabilities and legal implications. Participants also discussed potential role for BIM service providers, model managers, application vendors, cloud computing and software licensing models, highlighting related concerns over lack of experience if any of these are to be involved in an impending construction project.

Design Review and Clash Detection: Participants acknowledged the need for agreed protocols to conduct design review using BIM models and capabilities such as clash detection. Participants reported resistance among some stakeholder groups to change design review norms that still follow traditional methods. Discussions reflect increased confidence in collocated workplaces for multi-disciplinary integration and conflict resolution such as 'Big Room' concepts.

Version Management: Discussions reveal concern towards potential conflicts that may arise from multiple files and versions of the model generated across the team and the design development phases, besides the concerns towards software versions. 
Data Organization and Management: Data explosion and information overload emerged as a common concern across many participants. The need for archiving, history traceability, and data security were emphasized. Hence, a shared understanding of data management processes is needed.

\subsection{Context Mental Model}

Context refers to the team situation and scenarios. The discussions about the context revolved around the team's culture and practices, and what the FGI participants thought determines a team's response to known and known scenarios, including changes in tasks, processes and so on. Following were identified as the critical factors associated with context mental models:

Work Culture and Practice: Adopting IDDS approach will require adopting new work culture and news ways of working, and shifting organizational cultures towards an integrated service system.

Project Phase: Team members need to have clear understanding of their roles, responsibilities and contributions to the integrated BIM model at different phases of the project. This includes clarity on the scope of the model and its usage across the different phases of the project.

Regulations and Standards: Building regulations and standards vary across the different geographical regions, which needs alertness and clarifications, especially when working on projects or teams distributed across geographical boundaries.

Project Requirements: Team members need a shared understanding of the project requirements and the scope and level of integrated design delivery applicable to a given project. Discussions indicate the need for critical assessment of the project requirements at the outset of the project. For example, does the scale of the project justify how the team is organized for integrated design and delivery? How to monitor and measure the progress of the project? How should the team respond to the emergent and unforeseen project requirements, and what does it mean for the IDDS practice?

\subsection{Competence Mental Model}

Competence refers to the team's capabilities, which is either augmented or constrained by technology, which is once again related to the level of technology integration and technical competence of the team. With respect to BIM based collaboration for IDDS, competence appeared frequently in the discussions, often tied closely with team member mental models, i.e. perception of the competence of the other collaborators.

Competence of Project Partners and Their Tools: Project managers need to map the available resources and capabilities distributed across the people and tools to identify potential clashes and lack of compatibility. For example, the team may have 
the right tools but they may not have competent or skilled users. Teams may have competent users but they may not have the right tools. Or, teams may have competent users as well as competent tools, but the chosen tools may not necessarily be compatible, reducing the overall competence. At the same time, team members not only need to know about their competencies but they also need to know their limitations, and that of the tools being used. Issues of data format and import/ export capabilities of the software need to considered at the project planning stage itself. FGI participants also raised concerns over the other capabilities of the information systems that include security; bandwidth; server capacity; visualization capabilities; integration capabilities; querying and archiving; usability and interface.

Capabilities to Learn and Train during the Projects: How the team is organized and what knowledge management practices the team adopts, including how they use collaboration platforms and information systems, will determine how much the team members can learn during the project. Efficient teams can build on the opportunity for social learning and team building to increase competence during the project.

\subsection{Team Member Mental Model}

Team member mental model refers to team members' beliefs about each other, which determines how the team members interact and communicate with each other, and how they collaborate and work as a group. FGI discussions revealed a concern among the participants over the general tendency of functional groups and disciplines to work in isolation. Lack of trust in accuracy and completeness of the information and models created by members of other disciplines was a concern.

Discussions emphasized that roles and responsibilities need to be clearly defined, and the implications for the changing roles and responsibilities in the changing work environment needs to be communicated. Participants reported lack of clarity with changing roles and responsibilities across various dimensions such as, does the new role require new skills, or, do the changing roles and responsibilities restructure the actor network and the social ties? The discussions indicated that, in general, the team members seek answers to one common question: what is in there for me, and why should I change? That is, team members consider the effort towards the changing roles and responsibilities as an investment that must be rewarding, and there must be a return on investment. Some of the questions that individuals and firms in multiorganizational collaboration consider as part of their assessment of the return on investment include: Is it worth investing in a new technology unless the returns from the project or the alliance cover it? Is the project valuable enough to invest resources in adopting and learning new ways of working?

The task, process, context, competence and team member mental models are found to be closely interrelated, with the different issues overlapping across different mental models. For example, skills acquisition is not only about the competence but also about what the individuals think of the team, and their roles and responsibilities towards the team. Further research is needed to build on this mapping and develop a framework than can be used for computational application. 


\section{Conclusion}

This research adopts the construct of mental models as the basis for establishing the parameters of team work, especially in context of integrated design and delivery teams in multi-organizational construction projects. Findings from the focus group interviews and face to face interviews with representatives of various stakeholder groups are analysed and categorised into issues related to task, process, context, competence and team member mental models. The empirical data suggests that there are overlaps across the different mental models and the key issues concerning efficient teamwork and project management. Among other issues it is evident that team members have a sense of measuring their investment in the project and the returns on the investment. This investment involves multiple parameters such as time, effort, money, and reputation. Further research is planned to investigate these issues and develop the framework. In particular, the primary question raised for further investigation is: what is the threshold point beyond which the critical factors combine such that investment in BIM technologies, tools, processes and skills is perceived justifiable by the different stakeholders?

\section{References}

1. ACIF - Australian Construction Industry Forum. Construction Forecasting (2010), http: //www.acif.com.au/construction-forecasting/ (accessed on May 15, 2010)

2. Love, P.E.D., Gunasekaran, A., Li, H.: Concurrent engineering: a strategy for procuring construction projects. International Journal of Project Management 16(6), 375-383 (1998)

3. CIB. CIB White Paper on IDDS "Integrated Design and Delivery Solutions". CIB Publication 328 (2010) ISBN: 978-90-6363-060-7

4. AIA. Integrated Project Delivery: A Guide (2007), http : / /www . aia . org / contractdocs / AIAS 077630 (accessed on May 15, 2010)

5. King, G., Meyer, K.: Service integration and co-ordination: A framework of approaches for the delivery of co-ordinated care to children with disabilities and their families. Child: Care, Health, and Development 32, 477-492 (2005)

6. Moore, D.R., Dainty, A.R.J.: Integrated project teams' performance in managing unexpected change events. Team Performance Management, 212-222 (1999)

7. Orlikowski, W.J., Hofman, J.D.: An Improvisational Model of Change Management: The Case of Groupware Technologies. Sloan Management Review 38(2), 11-21 (1997)

8. Nah, F.F., Lau, J.L., Kuang, J.: Critical factors for successful implementation of enterprise systems. Business Process Management Journal 7(3), 285-296 (2001)

9. Glendinning, C.: Breaking down barriers: Integrating health and care services for older people in England. Health Policy 65, 139-151 (2003)

10. Badke-Schaub, P., Neumann, A., Lauche, K., Mohammed, S.: Mental models in design teams: a valid approach to performance in design collaboration? CoDesign 3, 5-20 (2007)

11. Klimoski, R., Mohammed, S.: Team Mental Model: Construct or Metaphor? Journal of Management 20(2), 403-437 (1994)

12. Langan-Fox, J., Anglim, J., Wilson, J.R.: Mental models, team mental models, and performance: Process, development, and future directions. Human Factors in Ergonomics and Manufacturing 14(4), 331-352 (2004) 
13. Lim, B.-C., Klein, K.J.: Team mental models and team performance: A field study of the effects of team mental model similarity and accuracy. Journal of Organizational Behaviour 27, 403-418 (2006)

14. Rouse, W., Cannon-Bowers, J., Salas, E.: The role of mental models in team performance in complex systems. IEEE Transactions on Systems Man and Cybernetics 22, 1296-1308 (1992)

15. Anumba, C.J., Baugh, C., Khalfan, M.M.A.: Organizational structures to support concurrent engineering in construction. Industrial Management and Data Systems 102(5), 260-270 (2002)

16. Mohammed, S., Dumville, B.C.: Team Mental Models in a Team Knowledge Framework: Expanding Theory and Measurement across Disciplinary Boundaries. Journal of Organizational Behavior 22(2), 89-106 (2001)

17. Ancona, D.G., Caldwell, D.: Improving the Performance of New Product Teams. IEEE Engineering Management Review 35(4), 45 (2007)

18. Cannon-Bowers, J.A., Salas, E.: Teamwork competencies, The interaction of team member knowledge skills and attitudes. In: O’Neil, O.F. (ed.) Workforce Readiness, Competencies and Assessment, pp. 151-174. Erlbaum, Hillsdale (1997)

19. Cohen, S.G., Bailey, D.E.: What Makes Teams Work: Group Effectiveness Research from the Shop Floor to the Executive Suite. Journal of Management 23(3), 239-290 (1997)

20. Donaldson, L.: The Contingency Theory of Organizations. Sage Publications, Thousand Oaks (2001)

21. Wilde, D.J.: Teamology: The Construction and Organization of Effective Teams. Springer (2007)

22. Kunz, J.C., Levitt, R.E., Jin, Y.: The Virtual Design Team: A Computational Simulation Model of Project Organizations. Communications of the Association for Computing Machinery 41, 84-92 (1998)

23. Singh, V.: Computational Exploration of Modes of Social Learning and Team Mental Models. PhD Thesis: Faculty of Architecture, Design and Planning, The University of Sydney, Australia (2010)

24. Ericsson, K.A.: Protocol analysis in psychology. In: Smelser, N., Baltes, P. (eds.) International Encyclopedia of the Social and Behavioral Sciences, pp. 12256-12262. Elsevier, Oxford (2001) 